\title{
Self-Reported Health and Safety Awareness Improves Prediction of Level of Care Needs in Veterans Discharged From a Postacute Unit
}

\author{
Julija Stelmokas, PsyD, Linas A. Bieliauskas, PhD, Katherine A. Kitchen Andren, PhD, \\ Robert Hogikyan, MD, MPH, Neil B. Alexander, MD
}

\begin{abstract}
Objectives: To evaluate the differential value of a self-reported health and safety awareness measure relative to other medical, psychosocial, and cognitive factors in predicting level of care (LOC) needs after hospital discharge.

Design: Retrospective medical record review.

Setting: Community living center postacute care (CLC-PAC) unit at a Veterans Affairs hospital.

Participants: A total of 175 veterans admitted to the Veterans Affairs hospital or directly to the CLC-PAC from home.

Methods: Cognitive status was assessed with the Mini-Mental State Examination, Digit Span Backward subtest, Trail Making Test (Part B), and Hopkins Verbal Learning Test-Revised. Self-report of health and safety awareness was measured with the Independent Living Scales Health and Safety (ILS-HS) subscale. Additional demographic and admission-related variables were coded, along with medical comorbidity, with the Charlson Comorbidity Index and depression using the Diagnostic and Statistical Manual of Mental Disorders, 4th Edition, Text Revision Depression Checklist.

Main Outcome Measurements: Increased level of care was collected from social work and occupational therapy notes and defined as increased assistance with activities of daily living or nursing home placement comparing prehospitalization with CLC-PAC discharge.

Results: A total of $19 \%(n=34)$ of residents required increased LOC on CLC-PAC discharge. The ILS-HS was a significant predictor of increased LOC above and beyond age and Mini Mental Status Examination score; for each standard deviation decrease in ILS-HS, there was an increased likelihood of greater LOC (odds ratio 0. 54, 95\% confidence interval 0.35-0.83). Other neuropsychological tests (memory, executive functioning) did not significantly improve the model.

Conclusions: The inclusion of the ILS-HS to a standard cognitive screen (Mini Mental Status Examination) can improve prediction of increased LOC. Although select aspects of memory and executive functioning independently contribute to increased LOC prediction, the ILS-HS likely measures a unique aspect of cognitive functioning that may be specific to discharge planning needs in CLC-PAC residents.
\end{abstract}

Level of Evidence: II

\section{Introduction}

Postacute care (PAC) often is considered for hospitalized patients who may not be ready for home discharge because of medical complexities, rehabilitation needs, and/or psychosocial issues [1-3]. Although most PAC residents meet rehabilitation goals and return home, a proportion exhibit slow or poor recovery, necessitating an increased level of care (LOC) postdischarge, including nursing home placement or institutionalization [4]. However, most studies evaluating LOC needs postdischarge, including discharge destination, are limited to hospitalized older residents $[4,5]$, and only a few have included patients in PAC settings $[6,7]$. Within Veterans Affairs hospitals, even less is known about the factors that predict LOC needs in residents admitted for PAC in community living centers (CLCs).

In addition to medical and psychosocial variables, cognitive status is considered an important factor that 
can influence functional status in patients undergoing rehabilitation [8]. The majority of studies to date, including those conducted within PAC settings $[6,7]$, use brief cognitive screens (such as the Mini Mental Status Examination [MMSE]) to measure cognitive status, which generally measure global rather than specific functions and are largely insensitive to executive dysfunction [9] - a key factor that can influence level of supervision needs [10]. In a review of the cognitive correlates of functional status, Royall et al [11] found that global cognition and executive functioning were the strongest, albeit modest, predictors of functional capacity compared with other cognitive domains. However, it is unknown whether these or other cognitive domains also contribute to increased LOC needs post-PAC discharge and whether functional measures can improve prediction of LOC.

The Independent Living Scales Health and Safety (ILSHS) subscale [12] is used to assess aspects of higherorder cognition (eg, insight, awareness, planning) by asking the respondent to answer hypothetical situations of potentially hazardous health and safety situations. Therefore, the ILS-HS directly evaluates aspects of functional capability that are needed for discharge planning and may provide greater ecological utility for predicting LOC needs than standard cognitive tests alone.

Our main objective was to evaluate the value of the ILS-HS in predicting increased LOC relative to a series of predictors, including global and specific cognitive domains. We hypothesized that a multivariate model including standard predictors such as age and MMSE, ILS$\mathrm{HS}$, but not other neuropsychological tests (eg, measures of memory and executive functioning), would improve the prediction of LOC.

\section{Methods}

\section{Participants}

Participants were predominantly male (94\%), white (86\%) veterans who were either directly admitted from home or after hospitalization (in another hospital unit) to the CLC-PAC unit at a Midwestern Veterans Affairs hospital between 2012 and 2014. Exclusion criteria included individuals admitted to the CLC-PAC unit within the previous year who already had completed neuropsychological testing in the same CLC-PAC unit and/or were primarily admitted for end-of-life care. Additional exclusionary criteria included significant sensory (low vision/blind/deaf) or motor impairment that interfered with visual motor tasks (eg, severe tremor, apraxia, or contractures). Given the need to evaluate a range of cognitive abilities, residents with impaired cognitive scores (MMSE $<24$ ) were included as long as they participated in rehabilitative therapies. Of the 968 residents admitted between 2012 and 2014, approximately $175(18 \%)$ met inclusion criteria and completed the cognitive screening measures.

\section{Procedure}

All participants completed routine, structured cognitive screening conducted by trained research assistants. Results were reviewed by clinical psychology predoctoral interns and clinical neuropsychology postdoctoral residents who were supervised by a licensed clinical psychologist. Cognitive screens were completed relatively early during CLC-PAC admission (average time from admission to testing was 7.3 days). The parent study was approved by the local health care system institutional review board. All data were retrieved through medical record review; informed consent was not required as part of this retrospective study.

\section{Measures}

Several demographic and admission-related variables were collected, including age, educational achievement, length of stay at the CLC-PAC, preadmission living status (alone or with others), and primary reason for admission. Medical comorbidity was measured with the Charlson Comorbidity Index [13]. Depression status was assessed during the cognitive screen interview with the modified Symptom Checklist for Major Depressive Disorders [14], a clinician-administered checklist of the Diagnostic and Statistical Manual of Mental Disorders, 4th Edition, Text Revision [15] depression criteria.

\section{Neuropsychological Tests}

Mini-Mental State Examination [16]. The MMSE was used as a measure of global cognition; scores range from 0 to 30 (greater scores indicate better cognitive performance).

Digit Span Backward [17]. The Digit Span Backward subtest was used as a measure of working memory; scores range from 0 to 16 (greater scores indicate better cognitive performance).

Trail-Making Test, Part B (TMT-B) [18]. The TMT-B was used as a measure of executive functioning; scores range from 0 to 300 (greater scores indicate worse performance).

Hopkins Verbal Learning Test-Revised [19]. The delayed free recall (Trial 4) was used as a measure of delayed memory; scores range from 0 to 12 (greater scores indicate better cognitive performance).

\section{LOC Needs}

The ILS-HS [12] was used to determine LOC needs based on the patient's self-report of responses to health and safety awareness questions. Administration of the 
ILS-HS required the individual to identify and reason through hypothetical situations related to potentially hazardous health and safety situations (eg, If you didn't have a regular doctor and you needed medical help quickly, how could you get it?) and provide an appropriate response (a score of 0 indicates lack of understanding or plan of action, a score of 1 indicates an generally concrete understanding or limited appreciation for consequences, and a score of 2 indicates good awareness and actions; total scores range from 0 to 40, with greater scores indicating better performance). The ILS initially was developed to determine the extent to which older adults can complete activities of daily living and live independently. The ILS-HS subscale has demonstrated good construct validity in communitydwelling healthy older adults [20], possible dementia [21], and can predict civil competency over and above other cognitive measures in heterogeneous clinical groups [22].

\section{LOC Assessment}

Social work and occupational therapy (OT) CLC-PAC admission notes were reviewed to determine whether the resident was independent or received ADL assistance (eg, received assistance from home health aide, required help with daily tasks such as meal preparation or medication management) before their hospitalization. Prehospitalization LOC needs were self-reported by the resident, having retrospectively reported LOC needs before the hospitalization. OT and social work notes usually corroborated these prehospitalization LOC needs. These notes were the source of LOC needs on discharge based on whether additional services were provided to the resident at discharge (eg, family member to assist to driving or cooking) and to document the discharge location. LOC was coded as "increased" if the resident received additional assistance compared with preadmission levels to help with daily tasks or was discharged to a nursing home or assisted living facility postdischarge.

\section{Statistical Analysis}

All cognitive scores were transformed into $z$ scores based on the group mean to allow for standardized comparisons relative to LOC. Logistic regression was used to evaluate the impact of factors on the likelihood that participants returned to preadmission level status or required a higher LOC. The likelihood ratio test (LRT) was used to determine whether the added variable improved the model. The MMSE was added as the first cognitive variable, given that a primary aim was to evaluate the added utility of the ILS-HS and additional neuropsychological assessment instruments beyond global cognition in predicting increased LOC. Preliminary analyses were conducted to ensure no violations of test assumptions, including normality/linearity and multicollinearity (via the variance factor inflation). Variables that were significantly correlated $(P<.05)$ with increased LOC based on initial univariate analyses were included in the logistic regression model.

\section{Results}

On admission to the CLC-PAC, the majority of the 175 residents reported living at home without assistance $(n=137 ; 78 \%)$. Although a majority returned to preadmission LOC levels, about $19 \%(n=34)$ required an increased LOC postdischarge, and of these, about $5 \%$ $(n=9)$ were discharged to an assisted living facility or nursing home (see Table 1 for LOC pre/post CLC-PAC admission). Descriptive characteristics of the sample and results from the univariate analyses comparing those who returned to preadmission levels $(n=141)$ and those who required a greater LOC $(n=34)$ are provided in Table 2. With the exception of age, no significant differences between these groups were found in demographic/psychosocial variables, depression status, medical comorbidity, and several admission-related variables (length of stay, primary reason for admission). Poorer scores on all cognitive measures, with the exception of working memory, were found in the greater LOC group.

Applying these key univariate differences in age, MMSE, and cognition, logistic regression and LRT models are displayed in Table 3 . Model 1 containing age was statistically significant, $\chi^{2}(1, n=175)=11.15$ and indicated that older age increases the likelihood of greater LOC (odds ratio [OR] 1.06, 95\% confidence interval $[95 \% \mathrm{Cl}] 1.02-1.10)$. Model 2 containing age and MMSE was statistically significant $\chi^{2}(2, \mathrm{n}=175)=$ 19.20 and indicated that for each standard deviation increase in MMSE there was a reduced likelihood of higher LOC (OR 0.58, 95\% Cl 0.40-0.85); Model 2 was superior to Model 1 in predicting increased LOC (LRT 8.05, $\mathrm{df}[1], P<.01)$. Model 3 containing age, MMSE, and the ILS-HS was statistically significant, $\chi^{2}(3, \mathrm{n}=$ $175)=27.95$ and indicated that for each standard deviation increase in ILS-HS there was a reduced likelihood of greater LOC (OR 0. 54, 95\% Cl 0.35-0.83); Model 3 was superior to Model 2 in predicting increased LOC (LRT 8.75, df[1], $P<.01$ ). Adding other cognitive measures including TMT-B did not add significantly to the model prediction.

Table 1

Increased LOC relative to preadmission assistance level

\begin{tabular}{lll}
\hline & & Increased LOC \\
Assistance Level & Prehospitalization & Postdischarge \\
\hline Home no assistance & 137 & 32 \\
Home with assistance & 38 & 2 \\
Total $(\mathrm{N})$ & 175 & 34 \\
\hline
\end{tabular}

$\mathrm{LOC}=$ level of care. 
Table 2

Descriptive statistics and results of univariate analyses of residents by LOC $(\mathrm{N}=175)$

\begin{tabular}{|c|c|c|c|}
\hline & $\begin{array}{l}\text { No Change LOC } \\
(\mathrm{n}=141)\end{array}$ & $\begin{array}{l}\text { Increased LOC } \\
(\mathrm{n}=34)\end{array}$ & \\
\hline Variable & $\begin{array}{l}\text { Mean (SD)/ } \\
\text { Frequency }\end{array}$ & $\begin{array}{l}\text { Mean (SD)/ } \\
\text { Frequency }\end{array}$ & $P$ value \\
\hline Age, y & $64.41(10.65)$ & $71.32(11.15)$ & $<.001$ \\
\hline Education, y & $12.72(2.48)$ & $12.88(2.38)$ & .73 \\
\hline Not married & 90 & 23 & .68 \\
\hline $\begin{array}{l}\text { Living with others } \\
\text { preadmission }\end{array}$ & 76 & 16 & .68 \\
\hline $\begin{array}{l}\text { Primary reason for } \\
\text { admission }\end{array}$ & & & .60 \\
\hline Wound & 47 & 8 & \\
\hline Orthopedic & 39 & 8 & \\
\hline Other* & 25 & 9 & \\
\hline Treatment $^{\dagger}$ & 19 & 4 & \\
\hline Cardio/pulmonary & 8 & 3 & \\
\hline Stroke/neurologic & 3 & 2 & \\
\hline Length of stay & 34.49 (22.77) & $41.70(28.50)$ & .12 \\
\hline Positive depression & 19 & 8 & .15 \\
\hline Charlson Index & $2.74(2.20)$ & $3.47(2.45)$ & .96 \\
\hline MMSE (raw) & $27.50(2.33)$ & $25.44(3.40)$ & $<.001$ \\
\hline DSB (raw) & 6.82 (1.90) & 6.32 (1.95) & .17 \\
\hline TMT-B (raw) & $160.33(83.81)$ & 224.27 (79.76) & $<.001$ \\
\hline HVLT-R (raw) & $4.62(2.82)$ & $3.35(3.28)$ & .03 \\
\hline ILS-HS (raw) & $33.16(4.35)$ & $28.53(6.70)$ & $<.001$ \\
\hline
\end{tabular}

LOC = level of care; SD = standard deviation; MMSE = Mini Mental Status Examination; DSB = Digit Span Backward; TMT-B = Trail-Making Test, Part; HVLT-R = Hopkins Verbal Learning Test-Revised; ILS-HS = Independent Living Scales Health and Safety.

* "Other" group includes deconditioning/debility, exacerbation in chronic health condition, mental status changes.

† "Treatment" group includes dialysis or chemotherapy.

\section{Discussion}

CLC-PAC veterans present with a variety of complex medical and functional needs, and, based on the present study, almost $20 \%$ will require additional assistance postdischarge (ie, additional assistance at home or institutionalization). Consistent with our hypothesis, the ILS-HS improved prediction of increased LOC, even when we accounted for age and MMSE. The addition of specific aspects of executive functioning or memory did

Table 3

Multivariate models predicting increased LOC

\begin{tabular}{lrlll}
\hline Model & B (SE) & $P$ Value & OR & $95 \% \mathrm{Cl}$ \\
\hline 1 & & & & \\
$\quad$ Age & $0.06(0.02)$ & $<.001$ & 1.06 & $1.02-1.10$ \\
2 Age & $0.04(0.02)$ & $<.05$ & 1.05 & $1.00-1.09$ \\
$\quad$ MMSE & $-0.54(0.19)$ & $<.001$ & 0.58 & $0.40-0.85$ \\
3 & & & & \\
Age & $0.03(0.02)$ & .16 & 1.03 & $0.99-1.07$ \\
MMSE & $-0.42(0.21)$ & $<.05$ & 0.66 & $0.44-0.99$ \\
ILS-HS & $-0.62(0.22)$ & $<.001$ & 0.54 & $0.35-0.83$ \\
\hline
\end{tabular}

$\mathrm{LOC}=$ level of care; $\mathrm{SE}=$ standard error; $\mathrm{OR}=$ odds ratio; $\mathrm{Cl}=$ confidence interval; MMSE = Mini Mental Status Examination; ILS-HS = Independent Living Scales Health and Safety. not improve LOC prediction beyond the MMSE and the ILS-HS and suggests that the combination of these 2 measures (ie, MMSE and ILS-HS) evaluates specific aspects of cognition that are highly relevant to LOC needs postdischarge. These findings are consistent with previous studies on cognitive correlates of functional outcomes [11] and highlight the importance of measuring general cognitive status to inform discharge planning. These results also underscore the ecological validity of the ILS-HS as a functional capability measure in CLC-PAC settings, which represents a unique aspect of higherorder cognitive functioning that is not assessed routinely by cognitive screens and standard neuropsychological tests-namely awareness and judgement of health and safety behaviors.

Contrary to our hypothesis, living alone before admission to the CLC-PAC was not significantly associated with increased LOC. Nevertheless, social support is an important variable that may be specific to other rehabilitation outcomes, such as rate of functional recovery [6]. Although delayed memory and performance on the TMT-B predicted increased LOC needs in univariate analyses, the ILS-HS correlates with other cognitive abilities, including executive functioning [23], and likely accounts for the additional predicted variance in LOC.

Although the application of the ILS-HS in a veteran PAC cohort appears to contribute to LOC needs, these findings are based on retrospective chart review. Moreover, caregiver or surrogate verification of selfreported LOC before admission was not always available; thus, LOC at discharge was based on OT and social work notes. Results of future studies could be enhanced by including, prospectively, performance-based measures, objective interview, and/or informant-based methods of functional capability at both admission and discharge. Further evaluation also is needed to determine whether cognitive status predicts the need for increased assistance with specific activities of daily living or predicts the overall amount of home care assistance needed (eg, hours of home health aide or assistance from family or friends). Future prospective studies could determine whether the ILS-HS, as opposed to global cognitive (ie, MMSE) or other tests of specific cognitive attributes such as planning, abstract concept formation, sustained attention, and visual memory, best predict LOC needs. Finally, future research is needed to determine whether and how the ILS-HS is distinct from other functional measures, such as the Kohlman Evaluation of Living Scales (KELS [24]). Both measures include a health/safety subscale that include a few similar items; however, differences in the administration can make the KELS less challenging than the ILS (ie, the KELS-Safety and Health uses pictures/stimuli that may cue participants, as opposed to the ILS-HS, which requires spontaneous recall), which could make it less suitable to use with individuals with mild cognitive impairment. 


\section{Conclusions}

The inclusion of a self-reported health and safety measure (ILS-HS) to a standard cognitive screen (MMSE) can improve prediction of increased LOC in CLC-PAC veterans. Although select aspects of memory and executive functioning independently contribute to increased LOC prediction, the ILS-HS measures a unique aspect of cognitive functioning that may be specific to discharge planning needs in CLC-PAC residents.

\section{Acknowledgments}

We thank University of Michigan's Center for Statistical Consultation and Research for support with statistical analysis and our research assistants (Anjali Sood, Stephanie Wong, and Elizabeth Jones) for their support with data coding.

\section{References}

1. Kosasih JB, Borca HH, Wenninger WJ, Duthie E. Nursing home rehabilitation after acute rehabilitation: Predictors and outcomes. Arch Phys Med Rehabil 1998;79:670-673.

2. Dillingham TR, Yacub JN, Pezzin LE. Determinants of postacute care discharge destination after dysvascular lower limb amputation. PM R 2011;3:336-344.

3. Sacks GD, Lawson EH, Dawes AJ, Gibbons MM, et al. Which patients require more care after hospital discharge? An analysis of postacute care use among elderly patients undergoing elective surgery. J Am Coll Surg 2015;220:1113-1121.

4. Abrahamsen JF, Haugland C, Nilsen RM, Ranhoff AH. Three different outcomes in older community-dwelling patients receiving intermediate care in nursing home after acute hospitalization. J Nutr Health Aging 2016;20:446-452.

5. Campbell SE, Seymour DG, Primrose WR. A systematic literature review of factors affecting outcome in older medical patients admitted to hospital. Age Ageing 2004;33:110-115.

6. Seematter-Bagnoud L, Lecureux E, Rochat S, et al. Predictors of functional recovery in patients admitted to geriatric postacute rehabilitation. Arch Phys Med Rehabil 2013;94:2373-2380.

7. Young $Y$, Xiong K, Pruzek RM. Longitudinal functional recovery after postacute rehabilitation in older hip fracture patients: The role of cognitive impairment and implications for long-term care. J Am Med Dir Assoc 2011;12:431-438.
8. Everink IH, van Haastregt JC, van Hoof SJ, Schols JM, et al. Factors influencing home discharge after inpatient rehabilitation of older patients: A systematic review. BMC Geriatr 2016;16:5.

9. Kahokehr A, Siegert RJ, Weatherall M. The frequency of executive cognitive impairment in elderly rehabilitation inpatients. J Geriatr Psychiatry Neurol 2004;17:68-72.

10. Hart T, Millis S, Novack T, Englander J, et al. The relationship between neuropsychologic function and level of caregiver supervision at 1 year after traumatic brain injury. Arch Phys Med Rehabil 2003;84:221-230.

11. Royall DR, Lauterbach EC, Kaufer D, Malloy P, et al. The cognitive correlates of functional status: A review from the Committee on Research of the American Neuropsychiatric Association. J Neuropsychiatry Clin Neurosci 2007;19:249-265.

12. Loeb PA. ILS: Independent Living Scales Manual. San Antonio, TX: Psychological Corp. Harcourt Brace Jovanovich; 1996.

13. Charlson ME, Pompei P, Ales KL, MacKenzie CR. A new method of classifying prognostic comorbidity in longitudinal studies: Development and validation. J Chronic Dis 1987;40:373-383.

14. Kashani J, McKnew D, Cytryn L. Symptom checklist for major depressive disorders. Psychopharmacol Bull 1985;21:957-958.

15. Americian Psychiatric Association. Diagnostic and Statistical Manual of Mental Disorders. 4th ed., text rev. Washington, DC: Americian Psychiatric Association; 2000.

16. Folstein MF, Folstein SE, McHugh PR. "Mini-mental state." A practical method for grading the cognitive state of patients for the clinician. J Psychiatr Res 1975;12:189-198.

17. Wechsler D. WAIS-IV: Administration and Scoring Manual. New York: The Psychological Corporation; 2008.

18. Reitan RM, Wolfson D. The Halstead-Reitan Neuropsycholgical Test Battery: Therapy and Clinical Interpretation. Tucson, AZ: Neuropsychological Press; 1985.

19. Brandt J, Benedict RH. Hopkins Verbal Learning Test-Revised. Odessa, FL: Psychological Assessment Resources, Inc; 2001.

20. Baird AD, Solcz SL, Gale-Ross R, Blake TM. Older adults and capacity-related assessment: Promise and caution. Exp Aging Res 2009;35:297-316.

21. Baird A, Podell K, Lovell M, McGinty SB. Complex real-world functioning and neuropsychological test performance in older adults. Clin Neuropsychol 2001;15:369-379.

22. Quickel EJ, Demakis GJ. The Independent Living Scales in civil competency evaluations: Initial findings and prediction of competency adjudication. Law Hum Behav 2013;37:155-162.

23. Bell-McGinty S, Podell K, Franzen M, Baird AD, et al. Standard measures of executive function in predicting instrumental activities of daily livng in older adults. Int J Geriatr Psychiatry 2002;17: 828-834.

24. Kohlman-Thompson L. The Kohlman Evaluation of Living Skills. 3rd ed. Bethesda, MD: The American Occupational Therapy Association; 1992.

\section{Disclosure}

J.S. Veterans Affairs Ann Arbor Healthcare System Mental Health Service, 116B, 2215 Fuller Road, Ann Arbor, Ml 48105; Veterans Affairs Ann Arbor Geriatric Research Education and Clinical Center, Ann Arbor, Ml; Department of Psychiatry, University of Michigan Medical Center, Ann Arbor, MI. Address correspondence to: J.S.; e-mail: jstelmok@med.umich.edu

Disclosure: nothing to disclose

L.A.B. Veterans Affairs Ann Arbor Healthcare System Mental Health Service, Ann Arbor, Ml; Department of Psychiatry, University of Michigan Medical Center, Ann Arbor, Ml

Disclosures outside this publication: expert testimony, various attorneys, and insurance; travel/accommodations/meeting expenses unrelated to activities listed, institutional support for meetings
K.A.K.A. Veterans Affairs Ann Arbor Healthcare System Mental Health Service, Ann Arbor, Ml; Department of Psychiatry, University of Michigan Medical Center, Ann Arbor, MI

Disclosure: nothing to disclose

R.H. Veterans Affairs Ann Arbor Geriatric Research Education and Clinical Center, Ann Arbor, Ml; Department of Internal Medicine and Division of Geriatric and Palliative Medicine, The University of Michigan, Ann Arbor, MI Disclosure: nothing to disclose 
N.B.A. Veterans Affairs Ann Arbor Geriatric Research Education and Clinical Center, Ann Arbor, MI; Department of Internal Medicine and Division of Geriatric and Palliative Medicine, The University of Michigan, Ann Arbor, MI Disclosure: nothing to disclose

The first author (J.S.) was supported by the VA Advanced Fellowship in Geriatrics at VA Ann Arbor Healthcare System. The authors would like to acknowledge the support of VA Rehabilitation Research and Development and the Office of Research and Development of the Department of Veterans Affairs. The authors also acknowledge support of the National Institute of Health Claude Pepper Center (AG024824) and the Michigan Institute for Clinical and Health Research (UL1TR000433).

Submitted for publication September 13, 2016; accepted March 23, 2017. 